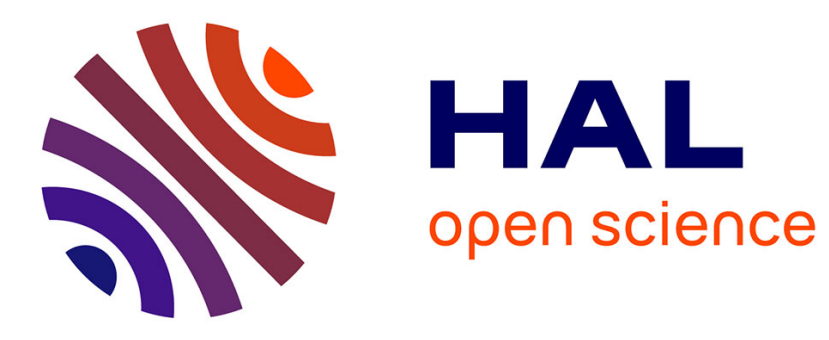

\title{
Mise en évidence de souches de Fusarium roseum var. sambucinum résistantes au thiabendazole, isolées à partir de tubercules de pomme de terre
}

Bernard Tivoli, Arnaud Deltour, Dominique Molet, Patrice Bedin, Bernard Jouan

\section{To cite this version:}

Bernard Tivoli, Arnaud Deltour, Dominique Molet, Patrice Bedin, Bernard Jouan. Mise en évidence de souches de Fusarium roseum var. sambucinum résistantes au thiabendazole, isolées à partir de tubercules de pomme de terre. Agronomie, 1986, 6 (2), pp.219-224. hal-00884868

\section{HAL Id: hal-00884868 https://hal.science/hal-00884868}

Submitted on 1 Jan 1986

HAL is a multi-disciplinary open access archive for the deposit and dissemination of scientific research documents, whether they are published or not. The documents may come from teaching and research institutions in France or abroad, or from public or private research centers.
L'archive ouverte pluridisciplinaire HAL, est destinée au dépôt et à la diffusion de documents scientifiques de niveau recherche, publiés ou non, émanant des établissements d'enseignement et de recherche français ou étrangers, des laboratoires publics ou privés. 


\title{
Mise en évidence de souches de Fusarium roseum var. sambucinum résistantes au thiabendazole, isolées à partir de tubercules de pomme de terre
}

\author{
Bernard TIVOli, Arnaud DELTOUR $\left({ }^{*}\right)$, Dominique MOLET $\left({ }^{*}\right)$, Patrice BEDIN $\left({ }^{* *}\right) \&$ Bernard JOUAN \\ I.N.R.A., Station de Phytopathologie, Centre de recherches de Rennes, B.P. 29. F-35650 Le Rheu \\ (*) Fédération nationale des Producteurs de Plant de Pomme de terre, 14, rue Cardinal Mercier, F-75009 Paris \\ (**) Institut technique de la Pomme de terre, Saint-Rémy-l'Honoré, F-78680 Les Essarts-le-Roi
}

RÉSUMÉ

Depuis 1982, des pourritures sont apparues sur des tubercules de pomme de terre de semence traités préalablement par du thiabendazole aussi bien dans des lots français que dans des lots importés en France. Dans de nombreux cas, il a été possible d'isoler des souches de Fusarium roseum var. sambucinum fortement résistantes au thiabendazole.

La comparaison de l'efficacité de l'imazalil, homologué en France contre le Phoma, seul ou en association avec le thiabendazole et celle du thiabendazole dans une série d'expérimentations sur tubercules artificiellement ou naturellement contaminés montrent que :

- le thiabendazole possède une efficacité sur les souches sensibles mais n'en a aucune sur les souches résistantes ;

- l'imazalil réduit le taux de tubercules pourris qu'ils soient infectés par une souche sensible ou par une souche résistante ;

- l'association imazalil-thiabendazole renforce l'efficacité de l'imazalil seul sur les 2 types de souches.

Les mesures de surveillance de l'évolution de la flore pathogène responsable de pourriture (Fusarium sp. et Phoma sp.) sont envisagées dans la conclusion.

Mots clés additionnels : Solanum tuberosum, fongicides, pourritures.

Isolation of thiabendazole-resistant strains of Fusarium roseum var. sambucinum from potato tubers.

Since 1982, dry rots have appeared on seed-potato tubers treated with thiabendazole, both French and imported tubers being affected. Among the fungi (Fusarium sp. and Phoma sp.) isolated from the dry-rotted tubers, certain strains of $F$. roseum var. sambucinum were highly resistant to thiabendazole. Comparison of the efficacy of imazalil alone or in association with thiabendazole with that of thiabendazole alone showed, in many experiments with artificially or naturally infested tubers, that $: 1)$ thiabendazole was adequately effective on sensitive strains, but not on resistant strains ; 2) imazalil decreased the level of dry-rotted tubers with either type of strain ; 3) the imazalil-thiabendazole association increased the efficacy of imazalil on either type of strain. Means are discussed for monitoring the evolution of the phytopathogenic microflora responsible for dry rots (Fusarium sp. and Phoma sp.).

Additional key words : Solanum tuberosum, fungicides, dry rots.

\section{INTRODUCTION}

Les pourritures sèches du plant de pomme de terre durant la période de conservation sont dues à diverses espèces ou variétés de parasites appartenant aux genres Fusarium et Phoma (BOYD, 1972 ; JOUAN et al., 1974) qui pénètrent dans les tubercules à la faveur de blessures occasionnées lors de la récolte ou du calibrage du plant.
Ces parasites sont limités par des mesures prophylactiques d'ordre «cultural " (rotations, limitation des blessures, cicatrisation) mais aussi par des traitements chimiques. Le formol, longtemps préconisé contre les Fusarium (LANSADE, 1950) est actuellement peu utilisé, en raison de sa phytotoxicité et de son effet corrosif ; des fumigations à base d'aminobutane ou des micropulvérisations à base d'imazalil se sont avérées très efficaces contre la gangrène (GRAHAM \& 
HAMILTON, 1970 ; CAYLeY et al., 1981) mais ont un effet plus limité sur les parasites du genre Fusarium. Les fongicides de la famille des benzimidazoles (bénomyl et thiabendazole), actifs sur Phoma exigua sont également efficaces contre Fusarium roseum var. sambucinum, $F$. solani var. coeruleum et d'autres Fusarium (LEACH \& NIELSEN, 1975 ; TASCA \& STOIANOVICI, 1984) et ont permis de limiter considérablement les pertes en conservation.

Depuis 1982, des pourritures sont apparues sur des tubercules de semence traités préalablement par du thiabendazole aussi bien dans des lots français que dans des lots importés en France. Plusieurs hypothèses ont retenu notre attention: modification éventuelle de la formulation du fongicide, sous-dosage du produit par l'utilisateur ou bien apparition d'une population de souches résistantes de tel ou tel champignon. Après vérification, les 2 premières hypothèses ont pu, dans la majorité des cas, être écartées et nous nous sommes penchés sur la troisième.

LEROUX (1985) définit 2 types de résistance aux fongicides : " la résistance physiologique " " concernant des individus qui, en conditions contrôlées, sont inhibés par des concentrations en fongicide supérieures à celles affectant de manière similaire des individus sensibles », et la " résistance en pratique ». Cette dernière "s'observe lorsque, dans la nature, une perte d'efficacité significative est provoquée (totalement ou partiellement) par la présence d'individus résistants au fongicide appliqué ». Dans le cas des pourritures, la situation décrite précédemment s'apparente à cette $2^{e}$ situation.

Devant l'échec des traitements, les souches des différents champignons responsables de pourritures, isolés durant l'hiver 1983-1984 à partir de tubercules traités ou non, sont analysées pour leur comportement vis-à-vis du thiabendazole. L'étude porte sur un certain nombre de lots français et de lots importés. De plus, la recherche d'un traitement chimique susceptible de remplacer le thiabendazole est également entreprise.

\section{MATÉRIEL ET MÉTHODES}

\section{A. Le matériel végétal}

Les tubercules de pomme de terre sont prélevés dans les exploitations par les techniciens de la Fédération Nationale des Producteurs de Plant pour les lots français, et aux frontières, par les agents du Service de la Protection des Végétaux pour les lots importés.

Les souches des différents parasites sont recueillies par isolement microbiologique sur un milieu gélosé à base d'extrait de malt, à partir de lots de pomme de terre (traités ou non) présentant des pourritures naturelles ou sur des lots artificiellement blessés afin de révéler la présence des parasites, puis repiquées sur milieu contenant du thiabendazole. Les différentes souches isolées en France correspondant chacune à une exploitation différente sont répertoriées dans le tableau 1.

Sur les lots importés des Pays-Bas, République Fédérale d'Allemagne, Danemark, Suisse, Pologne, Autriche et Grande-Bretagne, il a été isolé :
TABLEAU 1

Résistance au thiabendazole chez les champignons responsables de pourriture sèche isolés à partir de tubercules (traités ou non au thiabendazole) produits en France.

Thiabendazole resistance of the fungi causing dry rot of French potato tubers treated or not with thiabendazole.

\begin{tabular}{|c|c|c|c|c|}
\hline & \multicolumn{2}{|c|}{ Lots non traités } & \multicolumn{2}{|c|}{ Lots traités } \\
\hline & $\begin{array}{l}\text { Nombre } \\
\text { de souches } \\
\text { isolées }\end{array}$ & $\begin{array}{l}\text { Nombre } \\
\text { de souches } \\
\text { résistantes }\end{array}$ & $\begin{array}{l}\text { Nombre } \\
\text { de souches } \\
\text { isolées }\end{array}$ & $\begin{array}{l}\text { Nombre } \\
\text { de souches } \\
\text { résistantes }\end{array}$ \\
\hline $\begin{array}{l}\text { F. roseum var. } \\
\text { sambucinum }\end{array}$ & 74 & 0 & 66 & 57 \\
\hline $\begin{array}{l}F . \text { solani var. } \\
\text { coeruleum }\end{array}$ & 59 & 0 & 0 & 0 \\
\hline $\begin{array}{l}F . \text { roseum } \\
\text { divers }\left({ }^{*}\right)\end{array}$ & 196 & 0 & 4 & 0 \\
\hline $\begin{array}{l}P . \text { exigua var. } \\
\text { foveata }\end{array}$ & 34 & 0 & 0 & 0 \\
\hline $\begin{array}{l}P . \text { exigua var. } \\
\text { exigua }\end{array}$ & 103 & 0 & 2 & 0 \\
\hline
\end{tabular}

$\left.{ }^{*}\right)$ Il s'agit de $F$. roseum var. arthrosporioides, $F$. roseum var. culmorum, $F$. roseum var. graminearum, $F$. roseum type X.

- 16 souches de $F$. roseum var. sambucinum,

- 14 souches de $F$. solani var. coeruleum,

- 42 souches de $F$. roseum divers,

- 5 souches de $P$. exigua var foveata,

- 22 souches de $P$. exigua var. exigua.

\section{B. Etude de la gamme de sensibilité de divers parasites}

Des études préalables, par repiquage d'isolats mycéliens de différentes souches sensibles de parasite sur milieu gélosé à base d'extrait de malt contenant différentes doses de thiabendazole, ont permis de situer la dose inhibant le développement mycélien de chaque champignon. Elle se situe à $1 \mathrm{mg} / 1$ pour $F$. roseum var. sambucinum, var. culmorum et var. graminearum, $2 \mathrm{mg} / \mathrm{l}$ pour les 2 variétés de $P$. exigua et $5 \mathrm{mg} / \mathrm{l}$ pour $F$. solani var. coeruleum et $F$. roseum var. arthrosporioides.

\section{Tests d'activité du thiabendazole}

Ces tests d'efficacité se situent à 2 niveaux.

\section{In vitro}

La recherche des souches résistantes s'effectue sur des milieux 5, 10, 50 et 100 fois plus concentrés en thiabendazole que la dose minimale inhibitrice de la croissance de chacun des parasites.

Les lectures, réalisées après 2 semaines d'incubation à $20^{\circ} \mathrm{C}$, consistent à noter la dose de fongicide entrâ̂nant une réduction du développement mycélien de 50 p. 100 par rapport au témoin sans inhibiteur et celle permettant une inhibition totale. 


\section{In vivo}

L'efficacité des produits est appréciée sur tubercules en conditions d'infection artificielle ou naturelle.

\section{a) En conditions d'infection artificielle}

On apprécie l'efficacité du thiabendazole, de l'imazalil ou d'une association des 2 produits par rapport à un témoin non traité. Ces produits sont appliqués, en micropulvérisation, aux doses mentionnées dans le tableau 2, sur des tubercules infectés par une ou plusieurs souches sensibles et résistantes du parasite.

Les tubercules, blessés artificiellement à l'aide d'une machine réalisant environ 12 blessures par tubercule (JOUAN \& MOUTON, comm. pers.) sont trempés dans un bain d'inoculum. Cette suspension est produite à partir du broyage d'une culture obtenue en milieu liquide (contenu d'une fiole de Roux de 4 semaines de culture pour $5 \mathrm{I}$ d'eau) ou en milieu gélosé $(500 \mathrm{ml}$ d'inoculum mélangé à $50 \mathrm{l}$ d'eau).

Après $24 \mathrm{~h}$ de ressuyage, les traitements sont réalisés et les tubercules sont stockés à $15^{\circ} \mathrm{C}$, directement ou après un séjour de 2 semaines à $4{ }^{\circ} \mathrm{C}$.

Ces essais sont réalisés en utilisant, selon le cas, 3 ou 4 répétitions de 50 ou 65 tubercules.

\section{b) En conditions d'infection naturelle}

Les observations sont réalisées dans des exploitations dans lesquelles des souches de $F$. roseum var. sambucinum résistantes au thiabendazole sont décelées. Les tubercules, naturellement infectés (cultivars " Darésa » et « José ») subissent, après blessures, des traitements par les fongicides étudiés précédemment. Ils sont traités par trempage (cas de "José 》) ou micropulvérisation (cas de «Darésa »). Ces essais sont réalisés sans répétitions sur des lots de 200 tubercules.

Après 2 mois à une température comprise entre 12 et $20^{\circ} \mathrm{C}$, le niveau de maladie est apprécié par dénombrement des tubercules malades.

\section{RÉSULTATS}

\section{A. Mise en évidence de souches de $F$. roseum var. sambucinum résistantes au thiabendazole}

\section{Souches isolées à partir de lots français}

538 souches des différents parasites responsables de pourritures ont été isolées (tabl. 1). La flore parasitaire des tubercules non traités présente un éventail des divers parasites aussi bien au niveau des espèces ou variétés de Fusarium qu'au niveau des 2 variétés de Phoma. A l'inverse, à partir de tubercules traités, la flore isolée est presque exclusivement constituée de $F$. roseum var. sambucinum (66 souches sur les 72 isolées).

Parmi les parasites isolés, seules certaines souches de $F$. roseum var. sambucinum présentent une résistance vis-à-vis du thiabendazole et uniquement lorsqu'elles ont été isolées à partir de tubercules traités. Elles présentent un niveau élevé de résistance : la dose entraînant une réduction de 50 p. 100 de la croissance des souches, se situe environ à $75 \mathrm{mg} / 1$ pour l'ensemble des souches et, à $100 \mathrm{mg} / \mathrm{l}$ (soit 100 fois la dose minimale inhibitrice), elles ont une croissance représentant environ 45 p. 100 de celle du témoin (fig. 1).

\section{Souches isolées à partir de lots importés}

Sur un nombre limité de lots, traités ou non, provenant de divers pays (voir chap. II), 99 souches de Fusarium sp. et de $P$. exigua sont analysées pour leur comportement vis-à-vis du thiabendazole (tabl. 2).

Comme précédemment, sur l'ensemble des souches, seules des souches de $F$. roseum var. sambucinum présentent une résistance vis-à-vis du produit de traitement ( 7 souches sur 16$)$. Ces souches issues de lots traités ou non présentent le même niveau de résistance que celles isolées en France.



Figure 1

Influence du thiabendazole sur la croissance des colonies de $\mathrm{F}$. roseum var. sambucinum sensibles ou résistantes au thiabendazole (exprimée par le diamètre moyen des colonies de 15 souches, en $\mathrm{mm}$, après $10 \mathrm{j}$ de culture).

Action of thiabendazole on the mycelial growth of strains of F. roseum var. sambucinum sensitive or resistant to thiabendazole - mean diameter of cultures of 15 strains after 10 days on malt agar.

_ souche sensible; sensitive strain.

souche résistante; resistant strain.

TABLEAU 2

Résistance au thiabendazole chez les champignons responsables de pourritures sèches isolés à partir de tubercules (traités ou non au thiabendazole) importés en France.

Thiabendazole resistance of the fungi causing dry rot of imported potato tubers treated or not with thiabendazole.

\begin{tabular}{lccccc}
\hline \hline & $\begin{array}{c}\text { F. roseum } \\
\text { var. } \\
\text { sambucinum }\end{array}$ & $\begin{array}{c}\text { F. solani } \\
\text { var. } \\
\text { coeruleum }\end{array}$ & $\begin{array}{c}\text { F. roseum } \\
\text { Divers }\left(^{*}\right)\end{array}$ & $\begin{array}{c}P \text {. exigua } \\
\text { var. } \\
\text { foveata }\end{array}$ & $\begin{array}{c}P \text { exigua } \\
\text { var. } \\
\text { exigua }\end{array}$ \\
\hline $\begin{array}{l}\text { Nombre de } \\
\text { souches isolées }\end{array}$ & 16 & 14 & 42 & 5 & 22 \\
\hline $\begin{array}{l}\text { Nombre de } \\
\text { souches résistantes }\end{array}$ & 7 & 0 & 0 & 0 & 0 \\
\hline \hline
\end{tabular}

(*) Il s'agit de $F$. roseum var. arthrosporioides, F. roseum var. culmorum, $F$. roseum var. graminearum, $F$. roseum type $\mathrm{X}$. 


\section{B. Essais d'efficacité de fongicides vis-à-vis des sou- ches résistantes de $F$, roseum var. sambucinum}

Le développement brutal de souches de $F$. roseum var. sambucinum résistantes au thiabendazole implique un réajustement rapide des moyens de lutte chimique contre ce parasite. Avant la commercialisation éventuelle de nouvelles molécules chimiques capables de limiter son développement ainsi que celui des autres Fusarium et du Phoma, il s'agit d'étudier si, parmi les matières actives existantes, certaines, seules ou en association, présentent un bon niveau d'efficacité.

On compare donc l'efficacité de l'imazalil (homologué contre le Phoma) seul ou en association avec le thiabendazole et celle du thiabendazole dans une série d'expérimentations sur tubercules artificiellement ou naturellement contaminés.

\section{Sur tubercules artificiellement contaminés}

L'efficacité des produits est appréciée, d'une part, de façon échelonnée pendant les 2 mois qui suivent le traitement et, d'autre part, à un moment donné, soit 3 mois après l'application des produits.

\section{a) Cinétique de la maladie lors des divers trai- tements}

Quinze jours après la contamination artificielle (fig. 2), la totalité des tubercules infectés par les souches sensible ou résistante de $F$. roseum var. sambucinum est pourrie. Le thiabendazole possède, dans cet essai, une certaine efficacité sur la souche sensible (il reste environ 40 p. 100 de tubercules sains) mais n'en a aucune sur la souche résistante.

L'imazalil réduit le taux de tubercules pourris qu'ils soient infectés par la souche sensible ou par la souche résistante ; l'efficacité est identique à celle du thiabendazole sur la souche sensible.

Enfin, l'association imazalil-thiabendazole renforce l'efficacité de l'imazalil seul, sur les 2 types de souches : après 2 mois de conservation, 10 p. 100 des

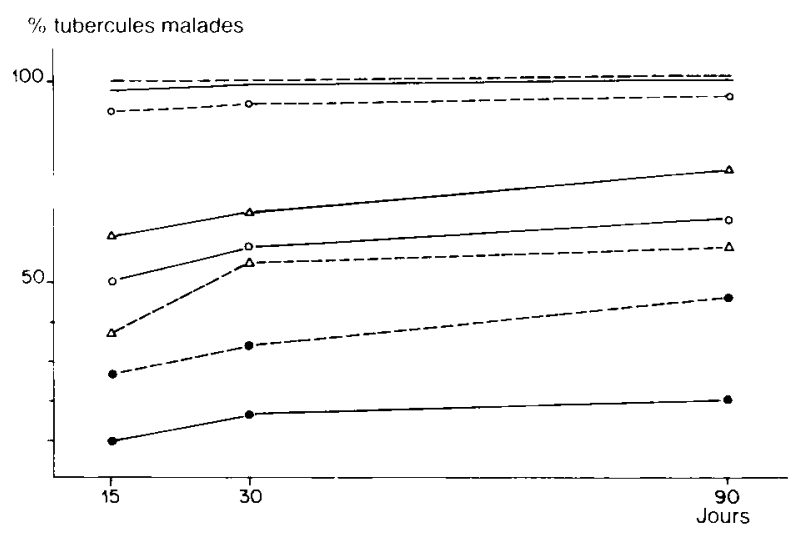

Figure 2

Comparaison de l'efficacité de fongicides sur des souches de F. roseum var. sambucinum sensibles ou résistantes au thiabendazole - exprimée par le pourcentage de tubercules malades.

Efficacy of fungicides on strains of $\mathrm{F}$. roseum var. sambucinum sensitive or resistant to thiabendazole.

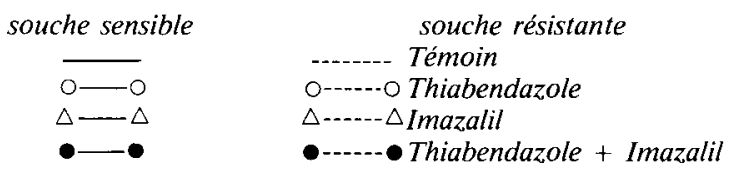

tubercules sont malades dans le cas de la souche sensible et 30 p. 100 dans le cas de la souche résistante.

De plus, ces résultats montrent clairement que le niveau des tubercules malades augmente dans le mois qui suit la contamination pour se stabiliser ensuite.

b) Appréciation de l'efficacité des produits, 3 mois après le traitement

Deux autres expérimentations sont réalisées avec 2 souches sensibles et 2 souches résistantes. L'appréciation du taux de tubercules malades 3 mois après la contamination confirme les résultats précédents (tabl. 3). Dans le cas des 2 souches sensibles, la réduction maximale de la maladie est obtenue dans le cas du thiabendazole, seul ou en association avec l'imaza-

TABLEAU 3

Efficacité de fongicides vis-à-vis de 2 souches de F. roseum var. sambucinum sensibles et résistantes au thiabendazole lors de 2 essais avec des tubercules infectés artificiellement - exprimée par le pourcentage de tubercules malades.

Efficacy of fungicides against 2 strains of $\mathrm{F}$. roseum var. sambucinum sensitive and resistant to thiabendazole in 2 experiments on artificially inoculated potato tubers (\% of dry-rotted tubers).

\begin{tabular}{|c|c|c|c|c|c|c|c|c|c|}
\hline \multirow{3}{*}{ Matière active } & \multirow{3}{*}{ Dose d'emploi } & \multicolumn{4}{|c|}{ Souches sensibles } & \multicolumn{4}{|c|}{ Souches résistantes } \\
\hline & & \multicolumn{2}{|c|}{ Souche 1} & \multicolumn{2}{|c|}{ Souche 2} & \multicolumn{2}{|c|}{ Souche 1} & \multicolumn{2}{|c|}{ Souche 2} \\
\hline & & Essai 1 & Essai 2 & Essai 1 & Essai 2 & Essai 1 & Essai 2 & Essai 1 & Essai 2 \\
\hline Imazalil & $30 \mathrm{~g} / \mathrm{t}$ & - & $14,2 \mathrm{a}$ & - & $30,7 \mathrm{abc}$ & - & $11,1 \mathrm{a}$ & - & 8,4 a \\
\hline $\begin{array}{l}\text { Imazalil }+ \\
\text { thiabendazole }\end{array}$ & $\begin{array}{l}15 \mathrm{~g} / \mathrm{t}+ \\
45 \mathrm{~g} / \mathrm{t}\end{array}$ & $2,3 a$ & $4,6 \mathrm{a}$ & $4,6 \mathrm{a}$ & $0,7 \mathrm{a}$ & $18,4 \mathrm{a}$ & $18,0 \mathrm{a}$ & $4,9 \mathrm{a}$ & $12,3 \mathrm{a}$ \\
\hline Thiabendazole & $60 \mathrm{~g} / \mathrm{t}$ & $7,3 \mathrm{a}$ & $11,5 \mathrm{a}$ & $11,1 \mathrm{ab}$ & $10,7 \mathrm{ab}$ & $31,1 \mathrm{ab}$ & 29,9 b & $36,5 \mathrm{ab}$ & $21,5 \mathrm{ab}$ \\
\hline Témoin non traité & 一 & $62,6 \mathrm{~b}$ & $61,5 \mathrm{~b}$ & $53,4 \mathrm{c}$ & $50,3 \mathrm{c}$ & $33,0 \mathrm{ab}$ & $29,9 \mathrm{~b}$ & $39,6 \mathrm{~b}$ & $31,5 \mathrm{~b}$ \\
\hline
\end{tabular}

Les valeurs de même signification (seuil de 5 p. 100) sont mentionnées par une lettre identique (a, b ou c).

Values not differing significantly at the $5 \%$ level are shown by the same letter ( $a, b$ or $c$ ). 
TABLEAU 4

Efficacité de fongicides vis-à-vis de souches résistantes de F. roseum var. sambucinum au thiabendazole dans le cas de tubercules naturellement infectés (exprimée par le pourcentage de tubercules malades).

Efficacy of fungicides against $\mathrm{F}$, roseum var. sambucinum resistant to thiabendazole on naturally infected potato tubers (\% of dry-rotted tubers).

\begin{tabular}{|c|c|c|c|c|}
\hline & \multicolumn{2}{|c|}{ Trempage (cultivar « José ») } & \multicolumn{2}{|c|}{ Micropulvérisation (cultivar « Darésa ») } \\
\hline & Dose matière active & $\begin{array}{l}\text { p. } 100 \text { de tubercules } \\
\text { malades }\end{array}$ & Dose matière active & $\begin{array}{c}\text { p. } 100 \text { de tubercules } \\
\text { malades }\end{array}$ \\
\hline Imazalil & $100 \mathrm{~g} / \mathrm{hl}$ & 4 & $一$ & - \\
\hline Imazalil + thiabendazole & $40+120 \mathrm{~g} / \mathrm{hl}$ & 9 & $15+45 \mathrm{~g} / \mathrm{t}$ & 3 \\
\hline Thiabendazole & $180 \mathrm{~g} / \mathrm{hl}$ & 27 & $60 \mathrm{~g} / \mathrm{t}$ & 25 \\
\hline Témoin non traité & eau & 40 & - & 40 \\
\hline
\end{tabular}

lil. Ce dernier fongicide, efficace sur la souche 1, l'est peu sur la souche 2 : respectivement 14 et 31 p. 100 de tubercules malades.

Sur les souches résistantes moins agressives dans ces essais que les souches sensibles, le thiabendazole présente très peu, voire aucune efficacité ; la meilleure protection est obtenue dans le cas de l'imazalil seul ou en association avec le thiabendazole.

\section{Sur tubercules naturellement infestés}

Aussi bien dans le cas du trempage que dans celui de la micropulvérisation, l'efficacité du thiabendazole est réduite: près de 25 p. 100 des tubercules sont encore malades. A l'opposé, l'imazalil seul ou en association avec le thiabendazole permet de réduire considérablement le taux de tubercules atteints de pourriture (tabl. 4).

\section{CONCLUSION}

D'après ces résultats, il apparaît que la résistance au thiabendazole affecte actuellement le seul $F$. roseum var. sambucinum aussi bien en France que dans d'autres pays. Cette résistance, de haut niveau, peut être à l'origine de réduction d'efficacité en pratique de traitements avec le thiabendazole.

Le fait que la résistance apparaisse d'abord chez $F$. roseum var sambucinum n'est sans doute pas un hasard : c'est l'espèce et la variété de Fusarium la plus fréquente et la plus agressive sur tubercules en voie de pourriture (TIVOLI \& JOUAN, 1981; TIVOLI et al., 1986) et, de plus, c'est un parasite dont l'aptitude à la sporulation est très élevée. Chez cette espèce, les risques de voir apparaître des mutants et de les sélectionner sont donc accrus.

Ces observations vont à l'encontre des travaux de BURTH et al. (1979) qui ont étudié in vitro les risques de voir apparaître des souches résistantes de $F$. solani var. coeruleum et de $F$. roseum var. sambucinum en repiquant ces parasites sur des milieux de culture contenant des doses croissantes de bénomyl et de thiabendazole. Au terme de leur étude, ils concluaient que les risques étaient faibles et que l'utilisation de ce type de produit pouvait se réaliser sans restriction particulière.

L'existence d'une parade dans la lutte chimique par l'utilisation d'imazalil permet de limiter avec une bonne efficacité l'extension du parasite en réduisant sensiblement les taux de pourriture. Son association avec le thiabendazole permet en plus de juguler efficacement les autres espèces ou variétés de Fusarium contre lesquelles l'imazalil seul n'est pas particulièrement efficace.

Il semble indispensable de renforcer la surveillance du comportement des autres parasites de conservation (Fusarium et Phoma) vis-à-vis du thiabendazole afin d'éviter de se trouver brutalement face à de nouveaux cas de résistance; cette surveillance systématique est actuellement effectuée en France par les laboratoires de la Fédération Nationale des Producteurs de Plant de Pomme de terre et par l'Institut National de la Recherche Agronomique.

Enfin, dans le but d'obtenir une meilleure connaissance des souches et, par conséquent, de concevoir une lutte plus efficace, l'étude de la biologie et de l'écologie des souches de $F$. roseum var. sambucinum résistantes au thiabendazole est actuellement entreprise en abordant notamment les problèmes d'agressivité, de compétition et de conservation des souches résistantes.

Reçu le 29 juillet 1985. Accepté le 18 octobre 1985.

\section{REMERCIEMENTS}

Les auteurs tiennent à remercier Mesdames Sailly, Chevalier \& M. LEMARChand (I.N.R.A., Rennes), TAVERNIER (F.N.P.P.P.T.) et M. Dusson (I.T.P.T.) pour leur participation active à ce travail ainsi que Monsieur Leroux (I.N.R.A., Versailles) qui a accepté de lire le document initial et d'y apporter des critiques constructives. 


\section{RÉFÉRENCES BIBLIOGRAPHIQUES}

Boyd A. E. W., 1972. Potato storage diseases. Rev. Plant Pathol., 51, 5, 297-321.

Burth V., Jahn M., Pett B., Gotz J., Brazda G., 1979. Möglichkeiten der Herausbildung resistenter Erreger bei der Kartoffelbeizung und ihre Bedeutung für das Beizverfahren. Arch. Phytopathol. Pflanzenschutz., Berlin, 15, 255-264.

Cayley G. R., Hide G. A., Tillotson Y., 1981. The determination of imazalil on potatoes and its use in controlling potato storage diseases. Pestic. Sci., 12, 103-120.

Graham D. C., Hamilton G. A., 1970. Control of potato gangrene and skin spot diseases by fumigation of tubers with sec-butylamine. Nature, 227, 297-298.

Jouan B., Lemaire J. M., Lemarchand E., Sanson M. T., 1974. Mise au point sur la gangrène ou «Phoma » de la pomme de terre, Phoma exigua var. exigua et var. foveata. Sci. Agron., Rennes, 97110.

Lansade M., 1950. Recherches sur la fusariose ou pourriture sèche de la pomme de terre, Fusarium coeruleum (Lib.) Sacc. Ann. Epiphyt., 1, 157-207.
Leach S. S., Nielsen L. W., 1975. Elimination of fusarial contamination on seed potatoes. Am. Potato J., 52, 211-218.

Leroux P., 1985. Les phénomènes de résistance des champignons phytopathogènes aux fongicides, t. 1, 19-45. Prem. Journées études maladies plantes, A.N.P.P., 22-27 février 1985, Versailles, $412 \mathrm{p}$.

Tasca G., Stoianovici I., 1984. Aspects of the efficiency of several fungicides applied after harvest on dry rot (Fusarium spp.) that occurs in storage of table potatoes. Potato Res., 27, 96.

Tivoli B., Jouan B., 1981. Inventaire, fréquence et agressivité des différentes espèces ou variétés de Fusarium responsables de la pourriture sèche des tubercules de pomme de terre. Agronomie, 1, 787 794.

Tivoli B., Abdul Razzaq K., Jouan B., Lemarchand E., 1986. Etude comparée des capacités infectieuses des différentes espèces ou variétés de Fusarium responsables de la pourritre sèche des tubercules de pomme de terre. Potato Res., 29 (sous presse). 